\title{
Direkte Demokratie im EU-Mehrebenensystem in Zeiten des Populismus
}

Annegret Eppler

\section{1. »Mehrebenendemokratie« in der Europäischen Union}

Das »europäische Mehrebenensystem ${ }^{1}{ }^{1}$ besteht aus der teils durch supranationales, teils durch intergouvernementales Regieren gekennzeichneten europäischen Ebene, aus den EU-Staaten mit ihren unterschiedlichen nationalen politischen Systemen und, wo vorhanden, aus ihren subnationalen politischen Systemen. ${ }^{2}$ Dieses europäische Mehrebenensystem ist eine "Mehrebenendemokratie", denn die miteinander verbundenen politischen (subnationalen, nationalen und supranationalen) Systeme sind demokratische Systeme, und das Mehrebenensystem verfügt über supranationale gemeinsame Legitimations-, Repräsentations- und direkte Partizipationskanäle ebenso wie über getrennte, an manchen Stellen verbundene und sich gegenseitig beeinflussende nationale, supra- und subnationale. Jedes der politischen Systeme bzw. jede - die supranationale, nationale und subnationale - der politischen Ebenen funktioniert nach einer eigenen Logik

1 Vgl. Wolfang Wessels, Das Politische System der EU (VS Verlag für Sozialwissenschaften, 2008); Johannes Pollak und Peter Slominski, Das politische System der EU (facultas, 2012); Thomas König, Elmar Rieger und Hermann Schmitt (Hrsg.), Das Europäische Mehrebenensystem: Mannheimer Jahrbuch für Sozialforschung Band 1 (hrsg. v. Vorstand des Mannheimer Zentrums für Europäische Sozialforschung, Campus, 1996).

2 Rudolf Hrbek, »Doppelte Politikverflechtung: Deutscher Föderalismus und die europäische Integration. Die deutschen Länder im EG-Entscheidungsprozess", in Rudolf Hrbek und Uwe Thaysen (Hrsg.), Die deutschen Länder und die Europäische Gemeinschaft (Nomos, 1986); Rudolf Hrbek und Sabine Weynand, Betrifft: Das Europa der Regionen. Fakten, Probleme, Perspektiven (C.H. Beck, 1994); Charlie Jeffery, »Towards a >Third Level in Europe? The German Länder in the European Union«, (1996) 44 Political Studies 253; Liesbet Hooghe, Gary Marks und Arjan Shakel, „Operationalizing Regional Authority: A Coding Scheme for 42 Countries, 1950-2006«, (2008) 18 Regional and Federal Studies 123; Jürgen Dieringer und Roland Sturm, Regional Governance in EU-Staaten (Barbara Budrich, 2010); Annegret Eppler und Fritz Staudigl, »Europeanisation of Austrian Federalism: The Case of Länder Rights in EU Affairs«, (2015) 24 Contemporary Austrian Studies 86. 
und verfügt über eigene Akteure, Institutionen, Normen, Prozesse und Verfahren. Das EU-Mehrebenensystem weist in vielerlei Hinsicht, aber nicht durchgehend, hierarchische Beziehungen im Sinne eines Verhältnisses der Über- und Unterordnung der politischen Ebenen zueinander auf. Diese »Demokratieverflechtung « geht mit einem hohen Maß an Komplexität und auch an Intransparenz einher. Legitimität, (repräsentative und direkte) Demokratie und Parlamentarismus erhalten jeweils eine spezifische, dem Mehrebenencharakter entsprechende Funktionslogik. Nicht nur die einzelnen demokratischen Systeme - d.h. das supranationale, die nationalen und die subnationalen - für sich, sondern auch das demokratische EU-Mehrebenensystem mit seinen demokratischen Subsystemen in ihrer Gesamtheit und insbesondere ihren Wechselwirkungen verdienen analytische Aufmerksamkeit.

Während die Veränderungen der Parlamente als »Kristallisationspunkte « der parlamentarischen Demokratien der verschiedenen politischen Systeme, insbesondere was ihre parlamentarischen Funktionen angeht, als »Europäisierungsphänomene« und ihre Kooperation (und auch Konkurrenz) als »Mehrebenenparlamentarismus« in den letzten Jahren ausführlich analysiert worden sind, ${ }^{3}$ finden sich neben einer Vielzahl verschie-

3 Andreas Maurer, »Perspectives de la coopération entre le Parlement européen et les Parlements nationaux", (Serie Politique W-19, Parlement européen, 1996); Andreas Maurer, Parlamentarische Demokratie in der Europäischen Union: Der Beitrag des Europäischen Parlaments und der nationalen Parlamente (Nomos, 2002); Andreas Maurer, "Mehrebenendemokratie und Mehrebenenparlamentarismus: Das Europäische Parlament und die nationalen Parlamente nach Lissabon«, in Stefan Kadelbach (Hrsg.), Europäische Integration und parlamentarische Demokratie (Nomos, 2009); Andreas Maurer, Parlamente in der EU (UTB/WUV, 2012); Arthur Benz, »Patterns of Multilevel Parliamentary Relations: Varieties and Dynamics in the EU and Other Federations«, (2017) Journal of European Public Policy 499; Annegret Eppler, »Vertikal und horizontal, bi- und multilateral: Interparlamentarische Beziehungen in EU-Angelegenheiten", in Gabriele Abels und Annegret Eppler (Hrsg.), Auf dem Weg zum Mehrebenenparlamentarismus? Funktionen von Parlamenten im politischen System der EU (Nomos, 2011); Annegret Eppler und Andreas Maurer (Hrsg.), Die Koordination der österreichischen Europapolitik (Nomos, 2018); Gabriele Abels und Annegret Eppler (Hrsg.), Subnational Parliaments in the EU: Multi-Level Parliamentary System: Taking Stock of the Post-Lisbon Era (Studienverlag, 2015); Ursula Münch und Eike-Christian Hornig (Hrsg.), Direkte Demokratie: Analysen im internationalen Vergleich (Nomos, 2015); Ben Crum und John E. Fossum, »The Multilevel Parliamentary Field: A Framework for Theorizing Representative Democracy in the EU«, (2009) 1 European Political Science Review 249; Arthur Benz, »Linking Multiple Demoi: Inter-Parliamentary Relations in the EU«, (2011) 1 IEV Online, www.fernuni-hagen.de/imperia/md/content/rewi/iev/benziev-online $2011 \mathrm{nr}$ 1.pdf (abgerufen am 19. Juni 2017). 
dener Ansätze zur Analyse von direktdemokratischen Verfahren in der EU und den einzelnen EU-Staaten kaum Studien des Zusammenspiels und der Wechselwirkungen der verschiedenen direktdemokratischen Verfahren, die in vielen der ineinander geschachtelten politischen Systeme zu finden sind ${ }^{4}$ und unter denen für diesen kurzen Text sehr grob sämtliche partizipative, deliberative, direktdemokratische Akte, auch Referenden und Direktwahlen, verstanden werden sollen. In Zeiten der Politikverdrossenheit und "postparlamentarischer ${ }^{5}$ Herausforderungen oft als eine Art Allheilmittel beschworen, stellt sich in Zeiten des Populismus die Frage, ob und wann direkte Demokratie zu einer Art Brandbeschleuniger werden kann. ${ }^{6}$ Nicht nur von einem auf die Output-Legitimität fokussierten Standpunkt - gegenüber dem alle gebotene Skepsis gilt -, muss aus der Mehrebenen-Perspektive hinterfragt werden, welche von mehreren ineinander geschachtelten politischen und konstitutionellen Systemen der nationalen Mehrebenensysteme und des supranationalen EU-Mehrebenensystems (lokal, regional, national, supranational) inwiefern und auf welche Weise von direkter Demokratie auf den verschiedenen (anderen) Ebenen gestärkt bzw. geschwächt werden. Es fragt insbesondere, wie hierbei Interdependenzen zwischen den verschiedenen Ebenen verlaufen.

Inwiefern wirkt sich das BREXIT-Referendum 2016 auf das supranationale System der EU, auf das subnationale System Schottlands und auf andere nationale und subnationale Systeme im EU-Mehrebenensystem aus? Inwiefern erfolgte durch das Referendum in Katalonien 2017 eine Legitimierung bzw. Delegitimierung oder Stabilisierung bzw. Destabilisierung der politischen Systeme Kataloniens, Spaniens und der EU? Stärkte der

4 Vgl. Beater Kohler-Koch und Berthold Rittberger, »Charting Crowded Territory: Debating the Democratic Legitimacy of the European Union «, in Beater KohlerKoch and Berthold Rittberger (Hrsg.), Debating the Democratic Legitimacy of the European Union" (Rowman \& Littlefield Publishers, 2007); Erik Oddvar Eriksen und John Erik Fossum (Hrsg.), Democracy in the European Union: Integration Through Deliberation? (Routledge, 2000); Cristina Fraenkel-Haeberle et al. (Hrsg.), Citizen Participation in Multilevel Democracies (Brill, 2015).

5 Svein S. Anderson und Tom R. Burns, »The European Union and the Erosion of Parliamentary Democracy: A Study of Post-Parliamentary Governance«, in Svein S. Anderson und Kjell A. Eliassen (Hrsg.), The European Union: How Democratic Is It? (Sage, 1996).

6 Eike-Christian Hornig, Mythos direkte Demokratie: Praxis und Potentiale in Zeiten des Populismus (Barbara Budrich, 2017); Eckhard Jesse und Roland Sturm, "Die Herausforderungen des 21. Jahrhunderts", in Eckhard Jesse und Roland Sturm (Hrsg.), Demokratien des 21. Jahrhunderts im Vergleich. Historische Zugänge, Gegenwartsprobleme, Reformperspektiven (VS Verlag für Sozialwissenschaften, 2003). 
Wahlkampf zur Direktwahl des österreichischen Bundespräsidenten 2016 europaskeptische Tendenzen und »schlug« dies auf die EU-Ebene oder auf andere EU-Staaten »durch«? Schränkte es die EU-Handlungsfähigkeit auch der "gemäßigten« damaligen Regierungsparteien ein und wurde dadurch das EU-System als Ganzes eventuell tangiert?

In Zeiten "multipler Krisen« der EU, deren Kern von vielen in einer hinter der wirtschaftlichen, territorialen und politisch-institutionellen Integration herhinkenden "gesellschaftlichen Integration ${ }^{7}$ gesehen wird, stellt sich insbesondere die Frage, inwiefern direktdemokratische Verfahren in den EU-Staaten in der Lage sind, die EU zu stärken bzw. zu schwächen. Dazu werden im Folgenden in aller gebotenen Kürze zwei Thesen ausgearbeitet: Die erste befasst sich mit der Abhängigkeit zukünftiger (Des-)Integrationsprozesse der EU von Legitimitätsfragen, die zweite mit möglichen Auswirkungen direktdemokratischer Verfahren auf verschiedenen politischen Ebenen auf die EU-Ebene.

\section{EU-(Des-)Integration und Legitimität}

Hinsichtlich des Aufbaus und der Weiterentwicklung des politischen Systems der Europäischen Union erfuhren zwei heute wesentliche Zusammenhänge über Jahrzehnte hinweg wenig Aufmerksamkeit. Zum einen befindet sich auch das supranationale EU-Mehrebenensystem, wie alle nationalen Mehrebenensysteme, in einem dynamischen Ausbalancierungsakt zwischen self rule und shared rule 8 und es ist nicht allein mit einer ständig fortschreitenden Integration, sondern auch mit »desintegrativen« Phänomenen zu rechnen. Zum anderen hat sich gezeigt, dass die Annahme eines

7 Vgl. Annegret Eppler, Lisa Helene Anders und Thomas Tuntschew, »Europe's Political, Social, and Economic (Dis-)Integration: Revisiting the Elephant in Times of Crises«, in Institute for Advanced Studies (Hrsg.), Political Science Series. Working Paper (Institute for Advanced Studies, 2016).

8 Daniel J. Elazar, Exploring Federalism (Tuscaloosa, 1987); Carl J. Friedrich, »Föderalismus in Theorie und Praxis«, (1964) 5 Politische Vierteljabresschrift 154; Michael Burgess, Comparative Federalism: Theory and Practice (Routledge, 2006); Arthur Benz und Jörg Broschek (Hrsg.), Federal Dynamics: Continuity, Change, and the Varieties of Federalism (Oxford University Press, 2013); Ludger Helms, Annegret Eppler und David Willumsen, »Is There a `German Schook of Comparative Federalism? An Institutional Perspective«, (2017) Zeitschrift für vergleichende Politikwissenschaft 533; Annegret Eppler, " Dynamic Federalism Theories` as Theories of European Integration?» (ECPR General Conference, Prague, 10. September 2016). 
"permissive consensus «", d.h. einer Art stillschweigenden und an Details wenig interessierten »ermöglichenden Konsenses« der Bevölkerungen der EU-Staaten hinsichtlich weiterer Integrationsschritte seit den großen wirtschaftlichen, territorialen und politisch-institutionellen Integrationsschritten, die durch den Maastrichter Vertrag angestoßen wurden und in seiner Folge realisiert wurden, nicht mehr haltbar ist.

Ein Großteil der politikwissenschaftlichen EU-Forschung konzentrierte sich auf die Analyse und Erklärung von Dynamiken in Richtung »mehr Integration " - insbesondere auf die erheblichen Vertiefungs- und Erweiterungsschritte seit 1951, erst in letzter Zeit auf die gesellschaftliche und nur mäßig auf die ökonomische Integration - und ignoriert weniger erfolgreiche Integrationsphasen, wie etwa die in der Integrationsgeschichte wiederholt auftretenden Konsolidierungs- und Stagnationsphasen und auch integrative Fehl- bzw. Rückschläge. ${ }^{10}$ Das derzeitige "empirische Puzzle zeigt in der anhaltenden Krise jedoch gleichzeitig ablaufende integrative und desintegrative Prozesse, ${ }^{11}$ die es zu erklären gilt. Nicht nur »Föderalismustheorien ${ }^{12}{ }^{12}$ auch andere theoretische Ansätze gehen von einer Richtungsoffenheit der europäischen Integration aus: Neofunktionalisten wie Lindberg und Scheingold sowie Schmitter ziehen seit Anfang der 1970er Jahre explizit die Möglichkeit eines »spill-backs«13 bzw. einer - einzelne

9 Leon N. Lindberg und Stuart A. Scheingold, Europe's Would-Be Polity: Patterns of Change in the European Community (Prentice-Hall, 1970), 62.

10 Annegret Eppler, »Politikwissenschaftliche Erklärungsansätze für die Dynamiken im EU-Mehrebenensystem«, in Anna Gamper et al. (Hrsg.), Föderale Kompetenzverteilung in Europa (Nomos, 2016).

11 Vgl. Annegret Eppler und Andreas Maurer, »Die simmer engere Union der Völker Europas« im Spannungsfeld zwischen Integration, Gruppenbildung und Desintegration«, in Anton Pelinka (Hrsg.), Europa - Hoffnung und Feindbild (Wochenschau Verlag, 2016); Eppler, Anders und Tuntschew, »Europe's Political, Social, and Economic (Dis-)Integration« (Fn. 7).

12 Insbesondere Friedrich, "Föderalismus in Theorie und Praxis« (Fn. 8); Carl J. Friedrich, Trends of Federalism in Theory and Practice (Praeger NY, 1968); vgl. Annegret Eppler, »European Where Necessary, National Where Possible«. Vorschläge aus den Niederlanden und Großbritannien zur Reform der EU im Lichte dynamischer Föderalismustheorien«, in Rudolf Hrbek und Martin Große Hüttmann (Hrsg.), Renaissance des Föderalismus in Europa? (Nomos, 2016).

13 Leon N. Lindberg, »Political Integration as a Multidimensional Phenomenon Requiring Multivariate Measurement«, in Leon N. Lindberg und Stuart A. Scheingold (Hrsg.), Regional Integration: Theory and Research (Harvard University Press, 1971); Lindberg und Scheingold, Europe's Would-Be Polity (Fn. 9). 
Indikatoren der Integration betreffende - »disintegration « in Betracht. ${ }^{14}$ Helen Wallace prägt die Metapher eines "politischen Pendels«, das sie zwischen der Arena der EU-Staaten und der supranationalen EU-Arena hin- und herschwingen sieht, ${ }^{15}$ und Maurer und Wessels unterscheiden Phasen großer Integrationsschritte von anderen Phasen der Stagnation und Konsolidierung in einer treppenartigen Entwicklung. ${ }^{16}$ Seit den 1990er Jahren analysieren Europäisierungsansätze auch die Bedingungen eines Rückschritts der Europäisierung, d.h. der Beeinflussung der nationalen Systeme durch die EU (»retrenchment «) ${ }^{17}$, und seit 2009 untersucht der Postfunktionalismus den möglicherweise bremsenden und desintegrativen Einfluss eines »constraining dissensus « ${ }^{18}$. Es ist also anzunehmen, dass Integrations- und Desintegrationsprozesse gleichzeitig stattfinden können, Integration und Desintegration zwei Seiten einer Medaille bzw. zwei Pole eines Konzepts ${ }^{19}$ sind und dass ständige die gleichzeitigen Dynamiken im Spannungsfeld von Einheit und Vielfalt, die ein Charakteristikum aller Mehrebenensysteme ist, auch im EU-System auftreten können. ${ }^{20}$ Integrati-

14 Lindberg, "Political Integration« (Fn. 13); Philippe C. Schmitter, "A Revised Theory of Regional Integration", in Lindberg und Scheingold (Hrsg.), Regional Integration (Fn 13). Vgl. auch den »dialektischen Neofunktionalismus« von Dorette Corbey, "Dialectical Functionalism: Stagnation as a Booster of European Integration«, (1995) 49 International Organization 253 und Zoe Lefkofridi und Philippe C. Schmitter, »Transcending or Descending? European Integration in Times of Crisis«, (2015) 7 European Political Science Review 3; Phillippe C. Schmitter und Zoe Lefkofridi, »Neofunctionalism as a Theory of Disintegration«, (2016) 1 Chinese Political Science Review 1.

15 Helen Wallace, »The Policy Process: A Moving Pendulum«, in Helen Wallace, Mark A. Pollack und Alasdair R. Young (Hrsg.), Policy Making in the European Union (Oxford University Press, 2010).

16 Andreas Maurer und Wolfgang Wessels, »The European Union Matters: Analysing Two Arenas Over Time«, in Wolfgang Wessels, Andreas Maurer und Jürgen Mittag (Hrsg.), Fifteen into One? The European Union and its Member States (Manchester University Press, 2003).

17 Claudio M. Radaelli, »The Europeanization of Public Policy«, in Kevin Featherstone und Claudio M. Radaelli (Hrsg.), The Politics of Europeanization (Oxford University Press, 2003).

18 Liesbet Hooghe und Gary Marks, »A Postfunctional Theory of European Integration: From Permissive Consensus to Constraining Dissensus«, (2009) 39 British Journal of Political Science 1.

19 Gary Goertz, Social Science Concepts: A User's Guide (Princeton University Press, 2006).

20 Eppler, Anders und Tuntschew, »Europe's Political, Social, and Economic (Dis-)Integration« (Fn. 7). 
on und Desintegration werden hier als Prozesse verstanden, deren Ergebnisse mit den gleichen (Outcome-)Indikatoren messbar sind. ${ }^{21}$

Eine hinter der wirtschaftlichen, territorialen und politisch-institutionellen Integration zeitlich zurückgebliebene "gesellschaftliche Dimension" europäischer Integration, ${ }^{22}$ messbar etwa an der gemeinsamen Identität, Zustimmung zum EU-System, grenzüberschreitenden Beziehungen der Bürgerinnen und Bürger und grenzüberschreitendem politischen Interesse, ${ }^{23}$ wurde seit dem »Nein « der Dänen zum Maastrichter Vertrag als »EUDemokratiedefizit« diskutiert. Spätestens seit Ausbruch der europäischen Banken-, Währungs- und Finanzkrise wird die "gesellschaftliche Dimension" europäischer Integration nicht nur im Hinblick auf den Aufbau der supranationalen Demokratie im EU-System, sondern auch in ihrer möglichen Funktion als Bremse weiterer Integration wahrgenommen. ${ }^{24}$ Die Analyse der Integration als multidimensionalen Prozess ist nichts Neues, sie geriet lediglich durch die Konzentration auf Vertiefungs- und Erweiterungsschritte (d.h. auf die politisch-institutionelle und territoriale Dimension der Integration) bzw. spätestens seit dem »Paradigmenwechsel « der Integrationstheorie und dem Verständnis der europäischen Integration als "Zustand « Anfang der 1990er Jahre etwas in Vergessenheit. Frühe Forscher wie $\mathrm{Nye}^{25}$ und andere Vertreter des Neofunktionalismus, ${ }^{26}$ des Transaktionalismus ${ }^{27}$ und des Föderalismus ${ }^{28}$ legten ihrer Forschung ein multidimensionales Verständnis europäischer Integration zugrunde und leiteten gerade aus der ökonomischen und gesellschaftlichen Dimension

21 Ebd.; für die Integrationsforschung zuerst Lindberg, »Political Integration« (Fn. 13). Vgl. William H. Riker, Federalism - Origins, Operation, Significance (Little Brown, 1964); William H. Riker, "Federalism«, (1975) 5 Handbook of Political Science 93; Friedrich, „Föderalismus in Theorie und Praxis« (Fn. 8); Friedrich, Trends of Federalism (Fn. 12).

22 Eppler, Anders und Tuntschew, »Europe's Political, Social, and Economic (Dis-)Integration« (Fn. 7).

23 Lisa Helene Anders, Annegret Eppler und Thomas Tuntschew, »Europäische Integration: Zweidirektional und Mehrdimensional«, (2016) 39 Integration 198.

24 Hooghe und Marks, »A Postfunctional Theory« (Fn. 18).

25 Joseph S. Nye, »Comparative Regional Integration: Concept and Measurement«, 22 International Organization 855.

26 Ernst B. Haas, The Uniting of Europe: Political, Social, and Economical Forces, 1950-1957 (University of Notre Dame Press, 1958).

27 Karl W. Deutsch, Nationalism and Social Communication: An Inquiry into the Foundations of Nationality (M.I.T. Press, 1966).

28 Friedrich, »Föderalismus in Theorie und Praxis« (Fn. 8); vgl. Eppler, »Dynamic Federalism Theories« (Fn. 8). 
(d.h. aus der Integration von Märkten und Gesellschaften) Erklärungen für die Dynamik der politisch-institutionellen Dimension ab.

Hinsichtlich dieser "gesellschaftlichen Dimension" nimmt der Postfunktionalismus die Ablösung des "permissive consensus" durch einen "constraining dissensus « an. ${ }^{29}$ Integrationskrisen werden teilweise auf die - früher noch als integrationsfördernd eingeschätzte ${ }^{30}$ - »Politisierung « als "polarization of opinions, interests or values and the extend to which they are publicly advanced towards the process of policy formulation within the EU «31 bzw. auf einen fehlenden "federal spirit « ${ }^{32}$ zurückgeführt und die Bedeutung einer »europäischen Seele« unterstrichen. ${ }^{33}$ Im Hinblick auf Dynamiken des europäischen Integrationsprozesses - Integration, Desintegration und Reintegration - stellt sich also die Frage, ob und unter welchen Bedingungen Demokratie - auch direkte Demokratie - im supranationalen Raum aber auch in den einzelnen EU-Staaten und ihren subnationalen Einheiten - eine »Zug- oder Gegenkraft« der europäischen Integration sein kann. ${ }^{34}$

29 Hooghe und Marks, »A Postfunctional Theory« (Fn. 18).

30 Philippe C. Schmitter, »Three Neo-functional Hypotheses about International Integration «, (1969) 1 International Organization 161, 166.

31 Vgl. Pieter de Wilde, »No Polity for Old Politics? A Framework for Analyzing the Politicisation of European Integration«, (2011) 5 Journal of European Integration 559, 560; Timm Beichelt et al. (Hrsg.), Civil Society and Democracy Promotion (Palgrave Macmillan, 2014); Francis Cheneval, Sandra Lavenex und Frank Schimmelfennig, »Demoi-cracy in the European Union: Principle, Institutions, Policies«, (2015) 22 Journal of European Public Policy 1.

32 Michael Burgess, In Search of the Federal Spirit: New Theoretical and Empirical Perspectives in Comparative Federalism (Oxford University Press, 2012); vgl. Klaus von Beyme, Föderalismus und regionales Bewusstsein. Ein internationaler Vergleich (C.H.Beck, 2007); Erik Oddvar Eriksen und John Erik Fossum, »Democracy through Strong Publics in the European Union?«, (2002) 40 Journal of Common Market Studies 401.

33 Werner Weidenfeld, Europas Seele suchen: Eine Bilanz der europäischen Integration (Nomos, 2017); vgl. Werner Weidenfeld und Nina Rümelin (Hrsg.), Europäische Identität: Voraussetzungen und Strategien (Nomos, 2007).

34 Marcus Höreth und Dennis-Jonathan Mann, »Die Legitimitätsfrage als Zug- oder Gegenkraft im europäischen Integrationsprozess?", in Annegret Eppler und Henrik Scheller (Hrsg.), Zur Konzeptionalisierung europäischer Desintegration: Zug- und Gegenkräfte im europäischen Integrationsprozess (Nomos, 2013). 


\section{Mögliche Effekte direkter Demokratie im EU-Mehrebenensystem}

Die Funktionslogik nationaler Demokratien kann nicht ohne weiteres auf das komplexe Mehrebenensystem der EU mit seiner supranationalen Ebene und den Interdependenzen zwischen den verschiedenen politischen Ebenen übertragen werden, ${ }^{35}$ die sich in verschiedenen, teils eigenständigen, teils zusammenhängenden, sich gegenseitig tangierenden Input-Legitimationsketten, Repräsentationskanälen und Partizipationsforen widerspiegeln.

Die EU sieht in der Demokratie einen der Werte, auf die sie sich gründet (Art. 2 EUV). Diese Werte werden nicht erst durch den Unionsvertrag geschaffen, sondern gehören zu dem in der Präambel genannten "gemeinsamen Erbe ${ }^{36}$ Sie gelten für die supranationale Ebene und binden die Unionsorgane (vgl. insbesondere Art. 9-13 EUV), sind darüber hinaus aber »allen Mitgliedstaaten [...] gemeinsam« (Art. 2 Satz 2 EUV). Damit ist eine Definition für die Nationalstaaten verbunden, die Mitglied der EU sind. Folgerichtig gehört Demokratie zu den Kriterien, die von Beitrittskandidaten zwingend erfüllt sein müssen (1993 durch den Europäischen Rat in Kopenhagen als Voraussetzung für den Beitritt als »Kopenhagener Kriterien« formuliert, seit dem Amsterdamer Vertrag 1999 im Primärrecht verankert, heute in Art. 49 Abs. 1 EUV). Entwickelt sich ein EU-Staat nicht dem Demokratieprinzip gemäß, ${ }^{37}$ sieht der Vertrag Sanktionen vor (Art.7 EUV $)^{38}$ - jedoch kann die EU selbstverständlich keinen Einfluss auf die konkrete Ausformung der demokratischen Systeme der EU-Staaten, natürlich auch nicht auf die Frage des dortigen Verhältnisses der repräsentativen zur direkten Demokratie, nehmen.

Auf EU-Ebene wählen die Bürgerinnen und Bürger das Europäische Parlament, das als supranationales Parlament Teil der Legislative auf EUEbene ist, Art. 14 EUV. Die nationalen, von den Bürgerinnen und Bürgern der Mitgliedstaaten nach dem jeweiligen Wahlsystem gewählten Regierungen sind Teil einer zweiten - direkten, wenn auch längeren - Legitimationskette, die zum Rat und damit zu einem zum Europäischen Parlament

35 Maurer, Parlamente in der EU (Fn. 3), 37-40.

36 Rudolf Geiger, Daniel-Erasmus Khan und Markus Kotzur (Hrsg.), EUV/AEUV. Vertrag über die Europäische Union und Vertrag über die Arbeitsweise der Europäischen Union (C.H. Beck, 2010), Art. 2 Rn. 1.

37 Vgl. Michael Blauberger, »Europäischer Schutz gegen nationale Demokratiedefizite«, (2016) 44 Leviathan 280.

38 Vgl. auch Annegret Eppler, »Die >Rechtsstaatskrise der EU«: Verderben zu viele Köche den Brei?«, (2016) 63 Zeitschrift für Politik 406. 
teilweise in einem horizontalen Kontroll-Verhältnis stehenden Legislativund Exekutivorgan führt. Die nationalen Parlamente nehmen nicht nur durch die Wahl der Regierungen (in parlamentarischen Demokratien), sondern auch über auf die europapolitischen Aktivitäten ihrer Regierungen und über direkt auf die EU bezogene Mitwirkungs-, Kontroll- und Monitoringmöglichkeiten am supranationalen Regieren teil. Im unmittelbaren Bezug zur supranationalen Ebene, z.B. über die Kontrolle der Subsidiarität und Verhältnismäßigkeit, begründen sie eine zusätzliche, regierungsunabhängige Legitimationskette, die auch die zweiten Kammern ${ }^{39}$ einbezieht, die in vielen Fällen die Mitwirkungsrechte der subnationalen Ebene an EU-Angelegenheiten sichern..$^{40}$ Der Dreiklang von Europäischem Parlament, Rat und nationalen Parlamenten wird, je nach Ausprägung in spezifischen Akteurskonstellationen, unter Kategorien wie "multilevel parliamentary democracy«, "compound representation " oder "joint decision-making" geordnet und analysiert. ${ }^{41}$

Dazu kommen vermehrt partizipative und deliberative Verfahren auf EU-Ebene. Seit dem Vertrag von Lissabon in Art. 11 EUV niedergelegt, waren sie zuvor etwa durch das Weißbuch »Europäisches Regieren« (2001; Grundsätze der EU-Politik: Offenheit, Partizipation, Verantwortlichkeit, Effektivität; Kohärenz) und den »Plan D for Democracy, Dialogue and Debate« (2005) der Europäischen Kommission lanciert worden (vgl. auch das seit dem Maastrichter Vertrag bestehende Petitionsrecht beim Europäischen Parlament und Beschwerderecht beim Europäischen Bürgerbeauftragten seit 1995). Seit den 1990er Jahren zieht die Kommission betroffene Kreise vor neuen Initiativen beratend hinzu, führt Online-Konsultationen (z.B. "Your voice in Europe«) und Bürgerkonferenzen durch, auf denen »normale« Bürgerinnen und Bürger diskutieren und bei denen versucht

39 Sven Leunig, Handbuch Föderale Zweite Kammern (Barbara Budrich, 2009).

40 Eppler und Staudigl, »Europeanisation of Austrian Federalism: The Case of Länder Rights in EU Affairs«; Annegret Eppler, »Multi-Level Governance in Europe - The Implication of German Laender in the Development of the Lisbon Treaty and the Strengthening of the Regional Level in Europe«, (2008) The Hebrew University International Law Research Paper No. 09-08, https://papers.ssrn.com/sol3/ papers.cfm?abstract_id=1271283 (abgerufen am 09. April 2018).

41 Vgl. Benz, »Patterns of Multilevel Parliamentary Relations« (Fn. 3) mit weiteren Typisierungen; vgl. Johannes Pollak, Repräsentation ohne Demokratie: Kollidierende Systeme der Repräsentation in der Europäischen Union (Springer, 2007); Timm Beichelt, »Regierungen als quasi-parlamentarische Akteure: Die Rolle der nationalen Exekutiven im Mehrebenenenparlamentarismus«, in Gabriele Abels und Annegret Eppler (Hrsg.), Auf dem Weg zum Mehrebenenparlamentarismus? Funktionen von Parlamenten im politischen System der EU (Nomos, 2011). 
wird, die Meinungsbildungsprozesse auf nationaler und supranationaler Ebene zu verschränken. ${ }^{42}$ Durch den Lissabon-Vertrag wurde die »Europäische Bürgerinitiative« eingeführt, durch die eine Million Bürgerinnen und Bürger, die aus unterschiedlichen EU-Staaten kommen, die Kommission auffordern können, einen neuen Rechtsakt zu initiieren. ${ }^{43}$ Diskutiert wurde auch die Einführung EU-weiter Referenden über zentrale Integrationsschritte und die Direktwahl etwa des Kommissionspräsidenten. ${ }^{44}$

Mit diesen direktdemokratischen, partizipativen und deliberativen Verfahren wird die Hoffnung auf eine weitere Demokratisierung der EU verbunden, jedoch stecken sie in den Kinderschuhen und unterliegen ganz erheblichen Herausforderungen: Während es an einer homogenen Gesellschaft, die von vielen als Voraussetzung für direktdemokratische Verfahren gesehen wurde, auch in nationalen Systemen mangelt, steht die EU vor der Herausforderung, dass nicht Bürgerinnen und Bürger eines demos, sondern intermediär wenig verbundener "multipler demoi «45 an direktdemokratischen Verfahren teilnehmen. Würden direktdemokratische Verfahren allerdings dazu beitragen, nach und nach einen "civic demos " ${ }^{46}$ oder eine gemeinsame europäische Identität (neben der bestehenden nationalen) ${ }^{47}$ zu schaffen, könnte ein solcher Fortschritt der »gesellschaftlichen Dimen-

42 Gabriele Abels, Annegret Eppler und Jennifer Träsch, "Zum >Demokratiedefizit der EU - und wie es sich (nicht) abbauen lässt", (2010) 3 Der Bürger im Staat 256.

43 Vgl. Andreas Maurer und Stephan Vogel, Die Europäische Bürgerinitiative. Chancen, Grenzen und Umsetzungsmöglichkeiten (SWP-Studie, 2009); Abels, Eppler und Träsch, »Zum >Demokratiedefizit $<$ der EU« (Fn. 43).

44 Heidrun Abromeit, "Ein Vorschlag zur Demokratisierung des europäischen Entscheidungssystems«, (1998) 1 Politische Vierteljahresschrift 80; Michael Zürn, »Über den Staat und die Demokratie im europäischen Mehrebenensystem«, (1996) 1 Politische Vierteljahresschrift 27.

45 J.H.H. Weiler, »Does Europe Need a Constitution? Demos, Telos and the German Maastricht Decision«, (1995) 1 European Law Journal 219; Dieter Grimm, Braucht Europa eine Verfassung? (Carl Friedrich von Siemens Stiftung, 1995); Peter Graf Kielmansegg, »Integration und Demokratie«, in Markus Jachtenfuchs und Beate Kohler-Koch (Hrsg.), Europäische Integration (Springer, 2003); Benz, »Linking Multiple Demoi« (Fn. 3).

46 Weiler, »Does Europe Need a Constitution?« (Fn. 45).

47 Vgl. Andreas Føllesdal und Simon Hix, »Why There is a Democratic Deficit in the EU: A Response to Majone and Moravcsik«, (2005) No. C-05-02 European Governance Papers, www.connex-network.org/eurgov/pdf/egp-connex-C-05-02.p df (abgerufen am 18. April 2017); Andreas Føllesdal, »The Legitimacy Deficits of the EU«, (2006) 14 The Journal of Political Philosophy 441; Armin Schäfer, »Das Demokratiedefizit der Europäischen Union«, (2006) 34 Leviathan 350. 
sion« der europäischen Integration ${ }^{48}$ weitere Integrationsschritte auch in anderen Integrationsdimensionen ermöglichen.

Die nationale Ebene dieser Mehrebenendemokratie besteht aus ganz unterschiedlichen demokratischen Systemen. ${ }^{49}$ Die supranationalen gemeinsamen Repräsentationskanäle des Europäischen Parlaments und direktdemokratischer Partizipationsmöglichkeiten auf EU-Ebene stehen parallel zu nach EU-Staaten getrennten, über die Arbeit der demokratisch gewählten Regierungen im Rat und über direkte Einflussnahmemöglichkeiten der nationalen Parlamente verbundenen und sich auf vielfältige Art gegenseitig beeinflussenden, nationalen Kanälen. ${ }^{50}$

In den einzelnen EU-Staaten werden die verschieden ausgestalteten repräsentativen Demokratien auf unterschiedliche Arten durch diverse direktdemokratische Instrumente flankiert ${ }^{51}$ und eventuelle Ausweitungen dieser direktdemokratischer Verfahren in Politik und Wissenschaft diskutiert. Wo bereits in Zeiten der Politikverdrossenheit eine genaue Abwägung der denkbaren direktdemokratischen Instrumente, Beteiligungs- und Entscheidungs-Quoren, zur Entscheidung gestellten Inhalte etc. vonnöten war, stellen sich diese Herausforderungen in Zeiten des Populismus umso mehr. Denn direktdemokratische Verfahren tangieren in hohem Maße Fragen der Einflussnahmemöglichkeiten (der »Machtverteilung «) innerhalb eines politischen Systems und werden nicht umsonst von Parteien wie der Alternative für Deutschland, der Freiheitlichen Partei Österreichs, der niederländischen Partij voor de Vrijheid, dem französischen Front National (seit Juni 2018 Rassemblement National), der Fidesz-Partei in Ungarn ge-

48 Eppler, Anders und Tuntschew, »Europe's Political, Social, and Economic (Dis-)Integration « (Fn. 7); Giesela Riescher, Sabine Ruß und Christoph M. Haas, Zweite Kammern (Wissenschaftsverlag, 2010); Susanne Bachmann, »Die Dynamisierung des österreichischen Bundesrates in der EU-Politik und sein Zusammenwirken mit anderen europapolitischen Akteuren«, in Eppler und Maurer (Hrsg.), Die Koordination der österreichischen Europapolitik (Fn. 3).

49 Vgl. Arend Lijphart, Patterns of Democracy: Government Forms and Performance in Thirty-Six Countries (Yale University Press, 1999); Wolfgang Ismayr, Gesetzgebung in Westeuropa: EU-Staaten und Europäische Union (Springer VS, 2004); Heinrich Pehle und Roland Sturm, »Die Europäisierung der Regierungssysteme«, in Oscar W. Gabriel und Sabine Kropp (Hrsg.), Die EU-Staaten im Vergleich: Strukturen, Prozesse, Politikinhalte (VS Verlag für Sozialwissenschaften, 2008).

50 Pehle und Sturm, »Die Europäisierung der Regierungssysteme« (Fn. 49).

51 Fraenkel-Haeberle et al. (Hrsg.), Citizen Participation (Fn. 4); Münch und Hornig (Hrsg.), Direkte Demokratie (Fn. 3); Andreas Kost, Direkte Demokratie (Springer, 2008); Peter Massing (Hrsg.), Direkte Demokratie: Eine Einführung (Wochenschau Verlag, 2005); Peter Massing et al., »Direkte Demokratie«, (2005) 381 Politische Bildung 4; vgl. die Beiträge des vorliegenden Bandes. 
fordert. Ein herausragendes Beispiel nicht nur für die Instrumentalisierung direkter Demokratie, sondern auch für ihr Einflusspotential auf andere Ebenen des EU-Systems ist das am notwendigen Teilnahmequorum gescheiterte (50\% der Wahlberechtigten hätten abstimmen müssen, getan haben es nur 39,9\%) Referendum der ungarischen Regierung vom 2. Oktober 2016. Zur Abstimmung stand, ob sich Ungarn an den vom Ministerrat im September 2015 festgelegten Verteilungsschlüssel für Flüchtlinge aus Italien und Griechenland halten sollte. Es zielte also darauf ab, rechtmäßig gesetztes EU-Sekundärrecht außer Kraft zu setzen.

Globalisierung, Internationalisierung und europäische Integration nach dem Weltkrieg auch als Mittel der Friedenssicherung verstanden - können angesichts grenzüberschreitender Phänomene (Terrorismus, Finanzkrisen, Migrationsbewegungen) einen Reflex nach nationaler Kontrolle auslösen. "Politikverdrossenheit « und gewandelte Protestkulturen ${ }^{52}$ werden teilweise von populistischen Strömungen abgelöst und die EU mit ihren supranationalen Organen, Mehrheitsentscheidungen in Rat und Parlament steht im Fokus vieler dieser Bewegungen, was eine Politisierung und Polarisierung der Politik in den EU-Staaten entlang europapolitischer Themen zur Folge hat. Argumente, die in der Diskussion über direkte Demokratie gegen dieselbe vorgebracht werden, erhalten im Lichte direktdemokratischer Verfahren, die EU-Fragen tangieren, eine neue Resonanz: ${ }^{53}$ Angeführt wird, dass Entscheidungskompetenzen hinsichtlich der hochkomplexen EU-Politikgestaltung nur schwer und über einen längeren Zeitraum erworben werden können. In einem von Ausgleich und Package-Deals geprägten supranationalen (Multi-level)Governance-System $^{54}$ können Sachfragen schwer auf Ja-/Nein-Abstimmungen herunter gebrochen werden (wohl aber die ultimative Frage nach Mitgliedschaft oder Austritt). ${ }^{55}$ Zeitliche Abläufe werden in hohem Maße vom EU-Politikzyklus geprägt und können kaum mit langwierigen direkten Verfahren auf nationaler Ebene verschränkt werden. Die Möglichkeit kleiner,

52 Annegret Eppler, Patrick Wauters und Diane Whitehouse, »MEP 2025: Preparing the Future Work Environment for Members of the European Parliament", (Study, European Parliament Think Tank, 2012).

53 Vgl. Kost, Direkte Demokratie (Fn. 51); Massing, Direkte Demokratie (Fn. 51); Massing et al., »Direkte Demokratie« (Fn. 51).

54 Liesbet Hooghe und Gary Marks, Multi-Level Governance and European Integration (Rowman \& Littlefield Publishers, 2001).

55 Vgl. Pieter De Wilde, Anna Leupold und Henning Schmidtke, »Introduction: The Differentiated Politicization of European Governance«, (2016) West European Politics 3. 
populistischer Gruppen die Diskussionen vor direktdemokratischen Entscheidungen über simple Argumente zu beherrschen, kann - unterstützt durch »Medienpopulismus" - europaskeptischen Strömungen dienen, gerade angesichts der zwischen verschiedenen Bevölkerungsschichten sehr unterschiedlichen Haltungen gegenüber der EU. ${ }^{56}$ Die Aufmerksamkeit, die direktdemokratischen Entscheidungen auf nationaler Ebene in Öffentlichkeit und Medien zuteil wird, und ihre (vermeintlich) ${ }^{57}$ erhöhte InputLegitimität bringt eine verstärkte Responsivität des gesamten politischen Prozesses mit sich: Finden in einem System direktdemokratische Entscheidungen in einem gewissen Ausmaß statt, beziehen politische Repräsentanten auch in verwandten Sachfragen und auch vor und nach konkreten direktdemokratischen Entscheidungen den angenommenen Volkswillen in ihre Willensbildung ein (was, wenn auch in geringerem Maße, auch schon bei Wahlerfolgen populistischer Parteien und ihrem Einzug in sub- und nationale Parlamente der Fall ist). Dies gilt aufgrund ihres Wiederwahl-Interesses für nationale Regierungen eher als für die nationalen Parlamente mit ihrer sich auch auf die EU-Politik der Regierungen beziehenden und insbesondere durch Oppositionsparteien ausgeübte Kontrollfunktion (vgl. die Rolle des britischen Parlaments in den BREXIT-Verhandlungen), was aufgrund der Mitgliedschaft der Regierungen im Rat und ihrer Scharnierfunktion zwischen den nationalen politischen Systemen und der supranationalen EU-Ebene ${ }^{58}$ gerade bei wichtigen, Einstimmigkeit erfordernden Entscheidungen, etwa über weitere Integrationsschritte, blockierend wirken kann. Die fehlende "gesellschaftliche Dimension« der Integration, manifestiert in der Rückbesinnung auf nationale Grenzen, kann sich so auf die politisch-institutionelle und territoriale Dimension der EU-Integration bremsend auswirken.

Diese "Demokratieverflechtung " geht bereits bei Einbeziehung der supranationalen und nationalen Ebene mit einem hohen Maß an Komplexität und Intransparenz einher. Bei Einbeziehung der subnationalen Ebene steigert sich die Komplexität nur deswegen nicht ins Unendliche, weil die subnationalen Einheiten im Verhältnis zu den nationalen weniger - und weniger sanktionsbewährten - Einfluss besitzen und entsprechend weniger ins Gewicht fallen. In insgesamt 74 Regionen der EU-Staaten Belgien,

56 Vgl. Hooghe und Marks, »A Postfunctional Theory« (Fn. 18); Cheneval, Lavenex und Schimmelfennig, «Demoi-cracy in the European Union« (Fn. 31).

57 Vgl. Kost, Direkte Demokratie (Fn. 51); Massing, Direkte Demokratie (Fn. 51); Massing et al., "Direkte Demokratie« (Fn. 51).

58 Robert D. Putnam, "Diplomacy and Domestic Politics: The Logic of Two-Level Games«, (1988) 43 International Organization 88. 
Deutschland, Großbritannien, Italien, Österreich, Spanien, außerdem auf Madeira und den Åland Inseln werden subnationale Parlamente mit eigenen Legislativkompetenzen gewählt. ${ }^{59}$ In vielen Staaten existieren auf dieser Ebene mehr direktdemokratische Beteiligungsmöglichkeiten als auf der nationalen Ebene. ${ }^{60}$ In der europäischen Mehrebenendemokratie wirken also auch die unterschiedlichen Traditionen subnationaler demokratischer Systeme, ${ }^{61}$ ihre Sicht der EU und ihre Einflussnahme auf diese »through multiple territorial lenses « ${ }^{62}$ auf die Funktionsdynamik ein. Ebenso haben Rückkoppelungseffekte des Mehrebenenregierens Auswirkungen auf die subnationalen Demokratien (etwa Eliten- und Patronagebildung durch Mitwirkungsmöglichkeiten auf der höheren Ebene je nach Bestellung der zweiten nationalen Kammer, Rechte von Minderheiten und "Minderheiten in Minderheiten ", Genderfragen etc.). ${ }^{63}$ Das Unabhängigkeitsreferendum in Katalonien 2017 zeigt, dass (durch direkte Demokratie womöglich verstärkte) Sezessionsbestrebungen subnationaler Einheiten, selbst wenn sie mit dem Ziel einer eigenen EU-Mitgliedschaft der abspaltungswilligen Region verfolgt werden, das EU-System zunächst aufgrund der Einstufung als nationale und von EU-Einfluss unabhängige Angelegenheit wenig tangieren und nicht als Schritt in Richtung europäischer »territorialer Integration ${ }^{64}$ verstanden werden können. Innerhalb Spaniens kann beobachtet werden, wie zwei unterschiedliche, jeweils demokratisch legitimierte Ebenen eines nationalen Mehrebenensystems in gegensätzliche Richtungen

59 Abels und Eppler (Hrsg.), Subnational Parliaments in the EU (Fn. 3).

60 Fraenkel-Haeberle et al. (Hrsg.), Citizen Participation (Fn. 4); Münch und Hornig (Hrsg.), Direkte Demokratie (Fn. 3); Kost, Direkte Demokratie (Fn. 51); Massing, Direkte Demokratie (Fn. 51); Massing et al., »Direkte Demokratie« (Fn. 51); vgl. die Beiträge des vorliegenden Bandes.

61 John Loughlin, Subnational Democracy in the European Union: Challenges and Opportunities (Oxford University Press, 2000); John Loughlin, Frank Hendriks und Anders Lidström (Hrsg.), The Oxford Handbook of Local and Regional Democracy in Europe (Oxford University Press, 2010); Markus Freitag und Adrian Vatter, Demokratien der deutschen Länder (Barbara Budrich, 2008).

62 Eve Hepburn, "Small Worlds in Canada and Europe: A Comparison of Regional Party Systems in Québec, Bavaria and Scotland «, (2010) 20 Regional \& Federal Studies 527, 528.

63 Arthur Benz, »Föderalismus und Demokratie: Eine Untersuchung zum Zusammenwirken zweier Verfassungsprinzipien«, (2003) 57 polis 1; Arthur Benz und Sabine Kropp, »Föderalismus in Demokratien und Autokratien: Vereinbarkeiten, Spannungsfelder und Dynamiken«, (2014) 8 Zeitschrift für Vergleichende Politikwissenschaft. Comparative Governance and Politics 1.

64 Indikator wäre hier "number of member states", vgl. Eppler, Anders und Tuntschew, »Europe's Political, Social, and Economic (Dis-)Integration« (Fn. 7). 
arbeiten und welche Sprengkraft direktdemokratische Verfahren dabei haben können.

\section{Fazit}

Die europäische Integration war nach dem Zweiten Weltkrieg zunächst ein Elitenprojekt, die Hoffnung war, dass aus der wirtschaftlichen, territorialen und politisch-institutionellen Integration Schritt für Schritt gesellschaftliche Integration erwachsen würde. Einen »permissive consensus «65 annehmend, wurden immense Integrationsfortschritte in sämtlichen Dimensionen der Integration erzielt. Erst nach dem »Nein« der Dänen zum Maastrichter Vertrag 1992, dem andere Referenden folgten (Niederlande und Frankreich 2005 über den Verfassungsvertrag, Irland über den Lissabon-Vertrag 2008 und noch einmal 2009) gelangten die immense Wichtigkeit der gesellschaftlichen Integration der europäischen Integration in den Fokus der politischen und wissenschaftlichen Diskussion. Angesichts des zunehmenden Erfolgs europaskeptischer Parteien (niederländische Partij voor de Vrijheid, der französische Front National, die deutsche Alternative für Deutschland, die italienische Fünf-Sterne-Bewegung und die Lega, die Freiheitliche Partei Österreichs, die PiS - Recht und Gerechtigkeit in Polen, der Fidesz in Ungarn etc.) im Zuge der Banken-, Schulden, Finanzund Wirtschaftskrise und zunehmender Politisierung und Polarisierung von EU-Themen gelangte die Möglichkeit eines »legal overstretch « ${ }^{66}$ und "constraining dissensus ${ }^{67}$ ins Blickfeld der EU-Forschung.

In diesen »multiplen Krisen" der EU ist die Pionierleistung, die die EU mit der Demokratisierung im supranationalen Raum, mit dem Europäischen Parlament als einzig direkt gewähltem supranationalen Parlament und mit ihren Strategien, die demokratischen Systeme der EU-Staaten zu vereinen, vollbringt, etwas in den Hintergrund gerückt. Ein Ausbau direktdemokratischer Verfahren auf den verschiedenen ineinander verschachtelten, supra-, sub- und nationalen, teilweise hierarchisch zueinanderstehenden Ebenen des EU-Systems birgt die Möglichkeit, ja, die Gefahr, dass sich die einzelnen demokratischen Systeme des Mehrebenensystems zu

65 Lindberg, »Political Integration« (Fn. 13).

66 Daniela Schwarzer, »Integration und Desintegration in der Eurozone«, in Eppler und Scheller (Hrsg.), Zur Konzeptionalisierung europäischer Desintegration (Fn. 34).

67 Hooghe und Marks, "A Postfunctional Theory« (Fn. 18). 
»kollidierenden Systemen ${ }^{68}$ der direkten Demokratie und einem Hemmnis weiterer Integration entwickeln könnten.

In politisch herausfordernden Zeiten der Vergangenheit haben direktdemokratische Verfahren eher nicht zu einer Stabilisierung und Konsolidierung von Demokratien beigetragen. Die weitere Stärkung der verschiedenen EU-bezogenen Parlamentsfunktionen, insbesondere der Artikulations- und Repräsentationsfunktion, aber auch der Kontrollfunktion (ex-ante und ex-post) von Parlamenten auf allen Ebenen des EU-Systems - also des Europäischen Parlaments, der nationalen und subnationalen Parlamente - sowie die Förderung ihrer Zusammenarbeit und Wahrnehmbarkeit im Sinne eines "Mehrebenenparlamentarismus" sind, gerade in krisenhaften Zeiten, im Vergleich zu direktdemokratischen Verfahren womöglich die bessere Alternative.

68 Pollak über repräsentativ-demokratische Systeme. vgl. Pollak, Repräsentation obne Demokratie (Fn. 41). 
